\title{
Acoustoelectric effect in Graphene with degenerate energy dispersion
}

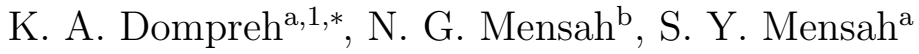 \\ ${ }^{a}$ Department of Physics, College of Agriculture and Natural Sciences, U.C.C, Ghana. \\ ${ }^{b}$ Department of Mathematics, College of Agriculture and Natural Sciences, U.C.C, Ghana
}

\begin{abstract}
The acoustoelectric effect $A E$ in Graphene with degenerate energy dispersion is theoretically studied for hypersound in the regime $q l>>1$. At low temperatures $\left(k_{\beta} T<<1\right)$, the non-linear dependence of Acoustoelectric current $j / j_{0}$ on the frequency $\omega_{q}$ and temperature $T$ are numerically analysed. The obtained graph for $j / j_{0}$ against $\omega_{q}$ qualitatively agreed with an experimentally obtained results. For $j / j_{0}$ versus $T$, the dependence of Acoustoelectric current in Graphene was found to manifest at low temperatures.

Key Words: Acoustoelectric effect, Graphene, Fermi-Dirac distribution

\section{Introduction}

In semiconducting materials, the need to acoustically generate d.c current for scientific applications has generated much interest. This phenomena is referred to as Acoustoelectric effect $A E$ and it involve the transfer of momentum from phonons to conducting charge carriers which leads to the generation of d.c. current in the sample. Acoustoelectric effect $(A E)$ in Bulk
\end{abstract}

\footnotetext{
${ }^{*}$ Corresponding author

Email: kwadwo.dompreh@ucc.edu.gh

Preprint submitted to Journal of ${ }^{A} T_{E} X$ Templates
} 
and Low-dimensional semiconducting materials has been extensively studied both experimentally [1, 2, 3, 4, 5, 6, 7] and theoretically [8, 9, 10, 11]. Recently, AE studies in Nano materials such as Graphene [12, 13, 14, 15, 16] and Carbon Nanotube (CNT) [17, 18, 19, 20] has attracted special attention. This is due to the remarkable electrical and mechanical properties of these materials especially the extreme electron mobility which persist at room temperatures. This makes Graphene and Carbon Nanotubes CNT suitable for applications in electronic systems such as light storage in quantum wells [21], generating single electrons [22] and photons, particularly for quantum information processing [23, 24, 25] and for inducing charge pumping in nanotube quantum dots. Experimentally, AE studies has been reported in Graphene [26, 27, 28, 29] but till date no theoretical analysis is reported. In this paper, the theoretical analysis of Acoustoelectric Effect $A E$ in Graphene in the hypersound regime $q l>>1$ (where $q$ is the acoustic wave number, $l$ is the mean free path of an electron) is carried out. The general expression is analysed numerically for various frequencies, and temperatures. The paper is organised as follows: In theory section, the theory underlying the Acoustoelectric effect in Graphene is presented. In the numerical analysis section, the final equation is analysed and presented in a graphical form. Lastly, the conclusion is presented in section 4 . 


\section{Theory}

We will proceed following the works of [13, 15], the Acoustoelectric Current in Graphene is given as

$$
\begin{array}{r}
j_{\overrightarrow{a c}}=-\frac{e \tau A\left|C_{q}\right|^{2}}{(2 \pi)^{2} V_{s}} \int_{0}^{\infty} k d k \int_{0}^{\infty} k^{\prime} d k^{\prime} \int_{0}^{2 \pi} d \phi \int_{0}^{2 \pi} d \theta\left\{\left[f(k)-f\left(k^{\prime}\right)\right] \times\right. \\
\left.V_{i} \delta\left(k-k^{\prime}-\frac{1}{\hbar V_{F}}\left(\hbar \omega_{q}\right)\right)\right\}
\end{array}
$$

with $k^{\prime}=k-\frac{1}{\hbar V_{F}}\left(\hbar \omega_{q}\right)$. For accoustic phonons, $C_{q}=\sqrt{|\Lambda|^{2} \hbar q / 2 \rho \hbar \omega_{q}}, \Lambda$ is the constant of deformation potential, $\rho$ is the density of the graphene sheet. $\tau$ is the relaxation constant, $V_{s}$ is the velocity of sound, and $A$ is the area of the graphene sheet. Here the acoustic wave will be considered as phonons of frequency $\left(\omega_{q}\right)$ in the short-wave region $q l>>1$ ( $q$ is the acoustic wave number, $l$ is the electron mean free path). The linear energy dispersion $E(k)= \pm \hbar V_{F}|k|$ (the Fermi velocity $V_{F} \approx 10^{8} \mathrm{~ms}^{-1}$ ) at the Fermi level with low-energy excitation. From Eqn.(1), the velocity $V_{i}=V\left(k^{\prime}\right)-V(k)$. Differentiating the energy dispersion yields

$$
V_{i}=\frac{2 \hbar \omega_{q}}{\hbar V_{F}}
$$

At low temperature $k_{B} T<<1$, the Fermi-Dirac distribution function become

$$
f(k)=\exp (-\beta(\varepsilon(k)))
$$

Inserting Eqn.(2) and Eqn.(3) into Eqn.(1) gives

$$
\begin{array}{r}
j_{\overrightarrow{a c}}=-\frac{2 A\left|C_{q}\right|^{2} \tau \hbar \omega_{q}}{(2 \pi)^{3} \hbar V_{F} V_{s}} \int_{0}^{\infty} k d k\left(k-\frac{1}{\hbar V_{F}}\left(\hbar \omega_{q}\right)\right)\left[\exp \left(-\beta \hbar V_{F} k\right)\right. \\
\left.-\exp \left(-\beta \hbar V_{F}\left(k-\frac{1}{\hbar V_{F}}\left(\hbar \omega_{q}\right)\right)\right)\right]
\end{array}
$$




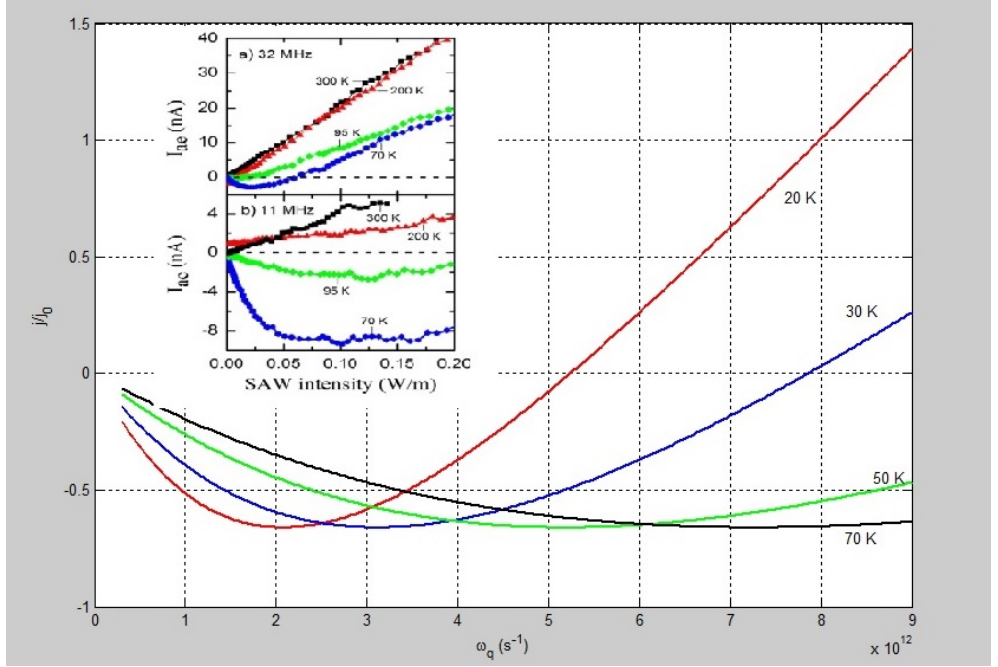

Figure 1: (a) Dependence of $j / j_{0}$ on $\omega_{q}$ for varying $T$. Insert: Dependence of Acoutoelectric Current $\left(I_{a e}\right)$ on SAW intensity for varying $T$ [27].

Using standard integrals in Eqn(4) and after a cumbersome calculation yields the Acoustoelectric Current $\left(j_{a c}\right)$ as

$$
j_{a c}=j_{0}\left\{2-\beta \hbar \omega_{q}\right\}\left[1-\exp \left(-\beta \hbar \omega_{q}\right)\right]
$$

where

$$
j_{0}=-\frac{2 \tau A|\Lambda|^{2} k T \hbar q}{(2 \pi)^{3} \beta^{3} \hbar^{4} V_{F}{ }^{4} \rho V_{s}}
$$

\section{Numerical Analysis}

The Eqn (5) is analysed numerically for a normalized graph of $j / j_{0}$ against $\omega_{q}$ and $T$. The following parameters were used $\Lambda=9 \mathrm{eV}, V_{s}=2.1 \times 10^{6} \mathrm{cms}^{-1}$ and $\vec{q}=10^{5} \mathrm{~cm}^{-1}$. In Figure 1, the graph for the dependence of $j / j_{0}$ on $\omega_{q}$ for varying $T$ is plotted. From the Figure (1), the non-linear graph of Acoustoelectric current $j / j_{0}$ decreases with an increase in temperature. The insert 

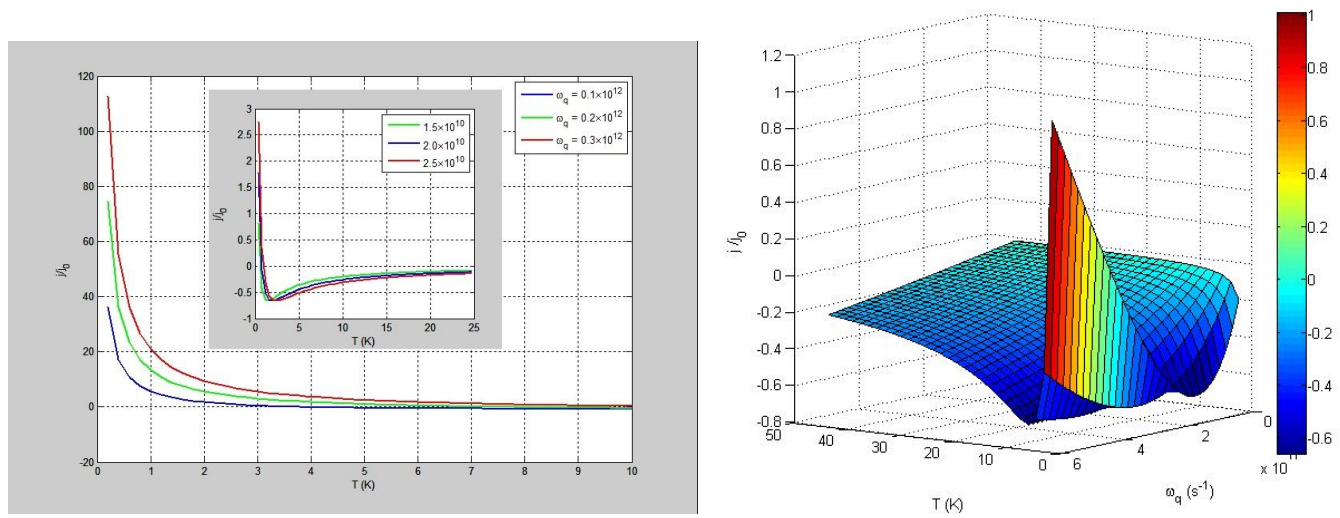

Figure 2: (a) Dependence of $j / j_{0}$ on temperature $T$ on varying $\omega_{q}\left(10^{10}\right)$, insert was plotted with $\omega_{q}\left(10^{12}\right)$, (b) A 3D graph of the dependence of $j / j_{0}$ on $\omega_{q}$ and $T$

is an experimentally obtained results of acoustoelectric current versus Surface Acoustic Wave (SAW) intensity. For acoustic phonons, the intensity is proportional to the frequency of the acoustic phonon i.e.I $=\hbar \omega_{q} f l u x$. Therefore, the theoretically obtained graph (see Fig. 1) qualitatively agrees with that obtained experimentally by Bandhu and Nash [27]. The acoustoelectric current $j_{a c}$ relates the hypersound absorption $\Gamma$ as

$$
j^{a c}=\frac{-2 e \tau}{\hbar V_{F}} \Gamma
$$

which is the Weinreich relation [31]. In Figure 2, the dependence of acoustoelectric current $j / j_{0}$ on temperature $T$ is plotted with varying $\omega_{q}$. At $\omega_{q}=10^{12}$, the acoustoelectric current decreases sharply to a minimum point and remain constant but at $\omega_{q}=10^{10}$ (see insert graph), the graph decreased pass the $j / j_{0}=0$ point to a minimum then raises to a constant values. For better understanding of the relation between $j / j_{0}, \omega_{q}$ and $T$, a 3D graph was plotted (See Figure 2b). In the Figure 2b, the maximum point point, 
$T=1.5 K, \omega_{q}=6 \times 10^{11} s^{-1}$ and $j / j_{0}=1.006$. At the minimum point, $T=1.5 K, \omega_{q}=1.2 \times 10^{11} s^{-1}$ and $j / j_{0}=-0.635$.

\section{Conclusion}

The Acoustoelectric effect in Graphene is studied in the hypersound regime $q l>>1$. At low temperatures, the theoretically obtained Acoustoelectric current $j / j_{0}$ qualitatively agreed with an experimentally obtained results.

\section{Bibliography}

[1] Gulayev, Yu V." Acousto-electric effect and amplification of sound waves in semiconductors at large sound intensities." Physics Letters A 30.4 (1969): 260-261.

[2] Ridley, B. K. "Nonlinear amplification of sound in a piezoelectric semiconductor." Journal of Physics C: Solid State Physics 3.4 (1970): 935.

[3] Gurevich, V. L., R. Katilius, and B. D. Laikhtman. "Nonlinear Amplification and Automodulation of Sound in Piezoelectric Semiconductors." Physical Review Letters 21.24 (1968): 1632.

[4] Gokhale, Vikrant J., Yu Sui, and Mina Rais-Zadeh. "Novel uncooled detector based on gallium nitride micromechanical resonators." SPIE Defense, Security, and Sensing. International Society for Optics and Photonics, 2012.

[5] Fleming, W. J., and J. E. Rowe. "Acoustoelectric effects in indium antimonide." Journal of Applied Physics 42.5 (1971): 2041-2047. 
[6] Ebbecke, J., et al. "Acoustic charge transport in GaN nanowires." Nanotechnology 19.27 (2008): 275708.

[7] Govorov, A. O., et al. "Nonlinear acoustoelectric transport in a twodimensional electron system." Physical Review B 62.4 (2000): 2659.

[8] Mensah,S. Y., Allotey, F. K. A., Mensah,N. G., Elloh,V. W., Amplification of acoustic phonons in a degenerate semiconductor superlattice. Physica E, Vol. 19(3) ,2003

[9] S. Y. Mensah, F.K.A. Allotey and S.K. Adjepong, J. Phys.: Condens. Matter 6, (1994) 6793

[10] S. Y. Mensah, F.K.A. Allotey and N.G. Mensah, J. Phys.: Condens. Matter 12, (2000) 5225

[11] S. Y. Mensah, F.K.A. Allotey and N.G. Mensah, H. Akrobotu, G. Nkrumah, Superlattice and Microstructure 37 (2005) 8797

[12] Ruvinskii, M. A., and B. M. Ruvinskii. "On the Hypersonic Absorption in Straight-line Graphene Ribbons." PHYSICS AND CHEMISTRY OF SOLID STATE 14, no. 4 (2013): 709-716.

[13] Dompreh, Kwadwo A., Natalia G. Mensah, and Samuel Y. Mensah. "Amplification of Hypersound in Graphene with degenerate energy dispersion." arXiv preprint arXiv:1503.07360 (2015).

[14] Dompreh, K. A., S. Y. Mensah, S. S. Abukari, F. Sam, and N. G. Mensah. "Amplification of Acoustic Waves in Graphene Nanoribbon in 
the Presence of External Electric and Magnetic Field." arXiv preprint arXiv:1410.8064 (2014).

[15] Nunes, O. A. C., and A. L. A. Fonseca. "Amplification of hippersound in graphene under external direct current electric field." Journal of Applied Physics 112, no. 4 (2012): 043707.

[16] Dompreh, K. A., S. Y. Mensah, S. S. Abukari, R. Edziah, N. G. Mensah, and H. Quaye. "Acoustomagnetoelectric Effect in Graphene Nanoribbon in the Presence of External Electric and Magnetic Field." arXiv preprint arXiv:1412.1678 (2014).

[17] Dompreh, K. A., N. G. Mensah, S. Y. Mensah, S. S. Abukari, F. Sam, and R. Edziah. "Hypersound Absorption of Acoustic Phonons in a degenerate Carbon Nanotube." arXiv preprint arXiv:1502.07636 (2015).

[18] Reulet, B., A. Yu Kasumov, M. Kociak, R. Deblock, I. I. Khodos, Yu B. Gorbatov, V. T. Volkov, C. Journet, and H. Bouchiat. "Acoustoelectric effects in carbon nanotubes." Physical Review Letters 85, no. 13 (2000): 2829-2832.

[19] Ebbecke, J., C. J. Strobl, and A. Wixforth. "Acoustoelectric current transport through single-walled carbon nanotubes." Physical Review B 70, no. 23 (2004): 233401.

[20] Dompreh, K. A., N. G. Mensah, S. Y. Mensah, F. Sam, and A. K. Twum. "Acoustoelectric Effect in degenerate Carbon Nanotube." arXiv preprint arXiv:1504.05484 (2015). 
[21] Cunningham, J., et al. "Acoustoelectric current in submicron-separated quantum wires." Applied Physics Letters 86.15 (2005): 2105.

[22] O. D. D. Couto, Jr., S. Lazic, F. Iikawa, J. A. H. Stotz, U. Jahn, R. Hey, and P. V. Santos, Nat. Photonics 3, 645 (2009).

[23] S. Hermelin, S. Takada, M. Yamamoto, S. Tarucha, A. D. Wieck, L. Saminadayar, C. Bauerle, and T. Meunier, Nature 477, 435 (2011).

[24] A. Hernandez-Minguez, M. Mouller, S. Breuer, C. Pfuuller, C. Somaschini, S. Lazic, O. Brandt, A. Garcia-Cristobal, M. M. de Lima, Jr., A. Cantarero, L. Geelhaar, H. Riechert, and P. V. Santos, Nano Lett. 12, $252(2012)$.

[25] Abergel, D. S. L., et al. "Properties of graphene: a theoretical perspective." Advances in Physics 59.4 (2010): 261-482.

[26] Miseikis, V., J. E. Cunningham, K. Saeed, R. ORorke, and A. G. Davies. "Acoustically induced current flow in graphene." Applied Physics Letters 100, no. 13 (2012): 133105.

[27] Bandhu, L. and Nash, G. R., Temperature dependence of the acoustoelectric current in graphene, Applied Physics Letters 105, 263106 (2014)

[28] Bandhu, L., L. M. Lawton, and G. R. Nash. "Macroscopic acoustoelectric charge transport in graphene." Applied Physics Letters 103, no. 13 (2013): 133101 .

[29] Miseikis, Vaidotas, John E. Cunningham, Kashif Saeed, Richard O’Rorke, and A. Giles Davies. "Acoustic charge transport in graphene." 
In Microwave Symposium Digest (MTT), 2012 IEEE MTT-S International, pp. 1-3. IEEE, 2012.

[30] Poole, Timothy, Lokeshwar Bandhu, and G. R. Nash. "Acoustoelectric photoresponse in graphene." Applied Physics Letters 106, no. 13 (2015): 133107.

[31] Weinreich G., 1956 Phys. Rev. 104321. 